\title{
Qualidade fisiológica de sementes de canola (Brassica napus L.) submetidas a microbiolização com Trichoderma spp.
}

\author{
Physiological quality of canola seeds (Brassica napus L.) submitted to \\ microbiolization with Trichoderma spp.
}

\section{Marisa Ana STRAHL'; Fabrício Penteado CARVALHO루 Emanuele JUNGES ${ }^{3}$; Cleudson José MICHELON ${ }^{4}$; Janaina Silva SARZI ${ }^{5}$; Marlove de Fátima Brião MUNIZ ${ }^{6}$}

\begin{abstract}
${ }^{1}$ Autor para correspondência: Eng. Agr., Mestranda em Agronomia (Produção vegetal), Universidade Federal de Santa Maria (UFSM), Departamento de Defesa Fitossanitária, Av. Roraima no 1000 - Faixa de Camobi, 97105-900, Santa Maria - RS, email:marisastrahl24@gmail.com

${ }^{2}$ Eng. Agr., Mestrando em Agronomia (Produção vegetal), Universidade Federal de Santa Maria (UFSM) - RS, e-mail:fcarvalho_sm@hotmail.com

${ }^{3}$ Docente do Instituto Federal Farroupilha - Campus São Vicente do Sul, São Vicente do Sul (IFFar) - RS, e-mail:emanuele.junges@iffarroupilha.edu.br

${ }^{4}$ Docente do Instituto Federal Farroupilha - Campus São Vicente do Sul, São Vicente do Sul (IFFar) - RS, e-mail:cleudson.michelon@iffarroupilha.edu.br

${ }^{5}$ Eng. Agr., Doutoranda em Agronomia, Universidade Federal de Santa Maria (UFSM) - RS, email:janainasarzi@yahoo.com.br ${ }^{6}$ Docente da Universidade Federal de Santa Maria (UFSM) - RS, email:marlovemuniz@yahoo.com.br
\end{abstract}

Recebido em: 16-08-2020; Aceito em: 17-05-2021

\section{Resumo}

O objetivo deste trabalho foi avaliar o efeito da microbiolização de sementes de canola (Brassica napus L.), com formulações comerciais à base de Trichoderma spp., sobre a qualidade fisiológica. Foram utilizados dois lotes de sementes de canola, de alta e baixa qualidade fisiológica, após realizou-se a microbiolização das sementes com diferentes formulações comerciais à base de Trichoderma spp. A qualidade fisiológica das sementes foi avaliada pelos testes de vigor através da primeira contagem de germinação, germinação, comprimento de parte aérea, comprimento de raiz, massa fresca de plântulas, massa seca de plântulas. O delineamento experimental utilizado foi o inteiramente casualizado, em fatorial $2 \times 5$ (Níveis de vigor $X$ produtos utilizados na microbiolização de sementes), totalizando 10 tratamentos, com quatro repetições cada. Houve interação significativa entre a microbiolização de sementes com Trichoderma spp. e a qualidade fisiológica de sementes de canola, para as variáveis de primeira contagem de germinação, germinação, comprimento de parte aérea e comprimento de raiz. Trichoderma spp. promoveu incremento na qualidade fisiológica no lote de sementes de canola com baixo vigor. Sementes de canola de baixo vigor, microbiolizadas com formulações comerciais a base de Trichoderma harzianum apresentam melhor qualidade fisiológica.

Palavras-chave adicionais: antagonismo; controle biológico; vigor de sementes.

\begin{abstract}
The objective of this work was to evaluate the effect of the microbiolization of canola seeds (Brassica napus L.), with commercial formulations based on Trichoderma spp., on the physiological quality. Two batches of canola seeds of high and low physiological quality, were used, after the microbiolization of the seeds was carried out with different commercial formulations based on Trichoderma spp. The physiological quality of the seeds was evaluated by vigor tests through the first count of germination, germination, shoot length, root length, fresh seedling weight, dry seedling weight. The experimental design used was completely randomized, in factorial $2 \times 5$ (Levels of vigor $\mathrm{X}$ products used in the microbiolization of seeds), totaling 10 treatments, with four repetitions each. There was a significant interaction between seed microbiolization with Trichoderma spp. and the physiological quality of canola seeds, for the variables of first germination count, germination, shoot length and root length. Trichoderma spp. promoted an increase in physiological quality in the canola seed lot with low vigor. Canola seeds of low vigor, microbiolized with commercial formulations based on Trichoderma harzianum present better physiological quality.
\end{abstract}

Additional keywords: antagonism; biological control; seed vigor.

Introdução

A canola (Brassica napus L.) constitui a segunda oleaginosa mais cultivada no mundo, atrás apenas da cultura da soja. Na safra 2018/2019, a produção global foi de 70,91 milhões de toneladas, sendo o Canadá, União Europeia e China os maiores produtores (USDA, 2019). A produção brasileira de canola, considerando o contexto internacional, é ainda bastante pequena. Com 35,5 mil hectares de área plantada, a 
produção nacional foi de 49,5 mil toneladas em 2018 , demonstrando a possibilidade de expansão. Os maiores produtores são os estados do Rio Grande do Sul e Paraná (CONAB, 2019). A cultura da canola vem sendo uma opção econômica, possibilitando alternativas à cobertura de solos ociosos durante o inverno, na entressafra do cultivo de milho e soja, podendo também diversificar áreas agrícolas, além de aumentar a renda para o agricultor (Tomm, 2007).

Em virtude do tamanho reduzido e da pequena quantidade de sementes usadas por hectare, é fundamental que sejam utilizadas sementes de elevada qualidade (Ávila et al., 2005). Por esta razão, a utilização de microrganismos através da microbiolização de sementes torna-se uma ferramenta inovadora para a cultura da canola, para que possa se obter uma maior qualidade fisiológica das sementes, além de proporcionar aumentos de produtividade da cultura.

A técnica de microbiolização é a aplicação de microrganismos via semente. Esta técnica tem sido utilizada com resultados satisfatórios e tem se apresentado como uma estratégia viável para a redução do uso exclusivo de produtos sintéticos, proporcionando benefícios econômicos e ambientais (Junges et al., 2014), promovendo benefícios na germinação de sementes, emergência e desenvolvimento das plântulas e produção de grãos e frutos (Harman, 2000).

A ação de microrganismos no desenvolvimento de plantas é ampla, incluindo os efeitos benéficos na germinação de sementes, emergência de plântulas, crescimento e produtividade de grãos. A utilização de promotores de crescimento de plantas pode ser uma alternativa viável para que ocorra a redução no uso de fertilizantes minerais, resultando no desenvolvimento de uma agricultura sustentável (Machado et al., 2012).

Fungos do gênero Trichoderma estão entre os principais microrganismos promotores do crescimento vegetal. A utilização deste fungo pode influenciar positivamente na germinação de sementes, no desenvolvimento, como também na produtividade da cultura, por produzir substâncias promotoras de crescimento e meIhoria na nutrição de plantas, principalmente pela solubilização de fósforo e síntese de ácido indol acético (Oliveira et al., 2012). Além de promotor de crescimento, os fungos do gênero Trichoderma também são capazes de atuar como agentes de controle de doenças de várias plantas cultivadas e indutores de resistência à doenças (Contreras et al., 2009; Santos et al., 2012; Silva et al., 2012; Asuming, 2013).

Além disso, as espécies de Trichoderma colonizam o sistema radicular, proporcionando o crescimento, o desenvolvimento e o aumento do número das raízes de plantas, facilitando a absorção e utilização de nutrientes, seguido do aumento da resistência aos estresses abióticos e consequentemente da produtividade das culturas (Kapri \& Tewari, 2010; Chagas et al., 2016 Chagas et al., 2017).

Diante disso, o objetivo do presente trabalho foi avaliar o efeito da microbiolização de sementes de canola, com formulações comerciais à base de Trichoderma spp., sobre a qualidade fisiológica de sementes de canola de alto e baixa vigor.

\section{Material e métodos}

O experimento foi conduzido no Laboratório de Sementes e Fitossanidade do Instituto Federal Farroupilha - Campus São Vicente do Sul, nos meses de outubro e novembro de 2019. Foram utilizadas sementes de canola (Brassica napus L.), cultivar Diamond, sem qualquer tipo de tratamento químico, com poder germinativo e vigor de $91 \%$, sendo o valor de vigor obtido pela primeira contagem de germinação, realizada aos cinco dias. Este foi considerado como Lote 1 de sementes de alta qualidade. $\mathrm{O}$ Lote 2 , de sementes de baixa qualidade, foi obtido através do envelhecimento acelerado do Lote 1. Para tanto, foram distribuídas 200 sementes sobre telas de alumínio, suspensas em caixas contendo $40 \mathrm{~mL}$ de água destilada, durante 24 horas à $42^{\circ} \mathrm{C}$ com $100 \%$ de umidade relativa $\cup R$ e, submetidas aos testes de germinação e vigor, seguindo as especificações descritas por Marcos Filho (1999). Após ser submetido às condições de envelhecimento acelerado, o Lote 2 apresentou $74 \%$ de vigor e $77 \%$ germinação, sendo o valor de vigor obtido pela primeira contagem de germinação, realizada aos cinco dias. Os lotes de sementes de alta e baixa qualidade compuseram os tratamentos avaliados submetidos ou não a microbiolização com Trichoderma spp.

Para a microbiolização das sementes dos dois Lotes, foram utilizadas as seguintes formulações comerciais: Trichodermil $\Theta$, Stimucontrol $\AA$, Ecotrich $\AA$ e Quality ${ }^{\circledR}$ (Tabela 1). Os tratamentos foram aplicados seguindo a recomendação dos fabricantes, em uma dose de $10 \mathrm{~mL}$ de Trichodermil $\circledast$ e Stimucontrol@ diluídos em $100 \mathrm{~mL}$ de água destilada e esterilizada para cada 100 sementes. Para Ecotrich $\AA$ e Quality $\AA$ foram utilizados $10 \mathrm{~g}$ de produto acrescido de $100 \mathrm{~mL}$ de água destilada e esterilizada para cada 100 sementes. No tratamento testemunha, para cada 100 sementes, foram adicionados $100 \mathrm{~mL}$ de água destilada e esterilizada. As sementes tratadas foram homogeneizadas suavemente em sacos plásticos e, em seguida, submetidas aos testes para avaliação de qualidade fisiológica.

As sementes tratadas foram colocadas sobre duas folhas de papel germiteste esterilizado, umedecido com água destilada e esterilizada, em caixas gerbox desinfestadas e cada caixa gerbox continha 25 sementes de $B$. napus, os testes foram instalados segundo as recomendações das Regras de Análise de Sementes (Brasil, 2009). Posteriormente, as caixas gerbox foram acondicionadas em estufa incubadora BOD (Biochemical Oxygen Demand), com temperatura constante de $25^{\circ} \mathrm{C}$ e fotoperíodo de 12 horas, segundo proposto por Brasil (2009) com adaptações. 
Tabela 1 - Formulações comerciais à base de Trichoderma spp. utilizadas no tratamento de sementes de canola (Brassica napus. L).

\begin{tabular}{llcc}
\hline Produtos & \multicolumn{1}{c}{ Espécie } & Concentração & Dose $/ 100$ sementes \\
\hline Stimucontrol & T. harzianum cepa CCT 7589 & $1,0 \times 10^{9} \mathrm{UFC} / \mathrm{L}$ & $10 \mathrm{~mL}(\mathrm{SC})^{*}$ \\
Ecotrich & T. harzianum - isolado IBLF006 & $1,0 \times 10^{10} \mathrm{UFC} / \mathrm{g}$ & $10 \mathrm{~g} \mathrm{(WP)}$ \\
Quality & T. asperellum & $1,0 \times 10^{10} \mathrm{UFC} / \mathrm{g}$ & $10 \mathrm{~g} \mathrm{(WG)}$ \\
Trichodermil & T. harzianum cepa ESALQ-1306 & $2,0 \times 10^{9}$ conídios viáveis $/ \mathrm{mL}$ & $10 \mathrm{~mL}(\mathrm{SC})$ \\
\hline
\end{tabular}

*SC: Suspensão concentrada; WP: Pó molhável; WG: Granulado Dispersível

O delineamento experimental utilizado foi o inteiramente casualizado, no arranjo fatorial $2 \times 5$ (Nível de vigor $\mathrm{X}$ produto para microbiolização de sementes), totalizando 10 tratamentos e quatro repetições.

A qualidade fisiológica das sementes foi avaliada quanto à:

- Primeira contagem de germinação realizada aos cinco dias após a instalação dos tratamentos.

- Germinação: contagem das plântulas normais germinadas aos sete dias após a instalação do teste.

- Comprimento de parte aérea e de raiz: retirou-se 10 plântulas aleatórias de cada tratamento, e as medições foram realizadas com paquímetro digital, ao sétimo dia, na última contagem realizada para germinação.

- Massa fresca e seca de plântulas: as plântulas utilizadas para determinar o comprimento foram levadas à secagem em estufa com ventilação de ar forçada a $65^{\circ} \mathrm{C}$ até peso constante. Ambas as pesagens foram feitas em balança de precisão.

Os dados foram analisados com comparação de médias entre os tratamentos pelo teste de Tukey, a $5 \%$ de significância. Para a análise estatística utilizouse o programa estatístico SISVAR versão 5.6 (Ferreira, 2008).

\section{Resultados e discussão}

Houve interação entre os fatores qualidade de semente e microbiolização com Trichoderma spp., para as variáveis primeira contagem de germinação, germinação, comprimento de parte aérea e comprimento de raiz. Para massa seca e fresca, a interação dos fatores não foi significativa (Tabela 2).

Tabela 2 - Qualidade fisiológica de lotes de sementes de canola (Brassica napus. L) de alto e baixo vigor, submetidas à microbiolização com bioprodutos comerciais à base de Trichoderma spp.

\begin{tabular}{|c|c|c|c|c|c|c|c|}
\hline \multicolumn{4}{|c|}{ Primeira contagem de germinação (\%) } & \multicolumn{4}{|c|}{ Germinação (\%) } \\
\hline \multirow{2}{*}{ Produto } & \multicolumn{2}{|c|}{ Qualidade da semente } & \multirow{2}{*}{ Média } & \multirow{2}{*}{ Produto } & \multicolumn{2}{|c|}{ Qualidade da semente } & \multirow{2}{*}{ Média } \\
\hline & $\mathrm{L} 1$ & $\mathrm{~L} 2$ & & & $\mathrm{~L} 1$ & L2 & \\
\hline Testemunha & $91,0 \mathrm{Aa}$ & $71,0 \mathrm{Bb}$ & $81,0 \mathrm{~B}$ & Testemunha & $91,0 \mathrm{Aa}$ & $75,0 \mathrm{Bb}$ & $83,0 \mathrm{~B}$ \\
\hline Ecotrich® & $91,0 \mathrm{Aa}$ & $85,0 \mathrm{Aa}$ & $88,0 \mathrm{AB}$ & Ecotrich® & $98,0 \mathrm{Aa}$ & $93,0 \mathrm{Aa}$ & $96,0 \mathrm{~A}$ \\
\hline Quality® & $94,0 \mathrm{Aa}$ & $80,0 \mathrm{ABb}$ & $87,0 \mathrm{AB}$ & Quality® & $96,0 \mathrm{Aa}$ & $86,0 \mathrm{Bb}$ & $91,0 \mathrm{~A}$ \\
\hline Trichodermil@ & $93,0 \mathrm{Aa}$ & $85,0 \mathrm{Aa}$ & $89,0 \mathrm{AB}$ & Trichodermil® & $94,0 \mathrm{Aa}$ & $90,0 \mathrm{Aa}$ & $92,0 \mathrm{~A}$ \\
\hline Stimucontrol® & $93,0 \mathrm{Aa}$ & $90,0 \mathrm{Aa}$ & $92,0 \mathrm{~A}$ & Stimucontrol $\AA^{\circledR}$ & $98,0 \mathrm{Aa}$ & $95,0 \mathrm{Aa}$ & $97,0 \mathrm{~A}$ \\
\hline Média & $92,0 \mathrm{a}$ & $82,0 \mathrm{~b}$ & & Média & $96,0 \mathrm{a}$ & $88,0 \mathrm{~b}$ & \\
\hline CV (\%) & & 7,3 & & CV (\%) & & 5,3 & \\
\hline \multicolumn{4}{|c|}{ Comprimento de parte aérea $(\mathrm{cm})$} & \multicolumn{4}{|c|}{ Comprimento de parte de raiz $(\mathrm{cm})$} \\
\hline \multirow{2}{*}{ Produto } & \multicolumn{2}{|c|}{ Qualidade da semente } & \multirow{2}{*}{ Média } & \multirow{2}{*}{ Produto } & \multicolumn{2}{|c|}{ Qualidade da semente } & \multirow{2}{*}{ Média } \\
\hline & L1 & $\mathrm{L} 2$ & & & L1 & $\mathrm{L} 2$ & \\
\hline Testemunha & $2,4 \mathrm{Ba}$ & $2,1 \mathrm{Bb}$ & $2,3 \mathrm{D}$ & Testemunha & $4,2 \mathrm{Aa}$ & $2,0 \mathrm{Bb}$ & $3,1 \mathrm{~B}$ \\
\hline Ecotrich $\AA$ & $2,7 \mathrm{Ba}$ & $2,9 \mathrm{Aa}$ & $2,8 \mathrm{AB}$ & Ecotrich® & $2,8 \mathrm{Ba}$ & $3,0 \mathrm{Aa}$ & $2,9 \mathrm{BC}$ \\
\hline Quality® & $2,6 \mathrm{Ba}$ & $2,2 \mathrm{Bb}$ & $2,4 \mathrm{CD}$ & Quality® & $2,2 \mathrm{Ba}$ & $2,3 \mathrm{ABa}$ & $2,3 \mathrm{D}$ \\
\hline Trichodermil® & $2,4 \mathrm{Bb}$ & $2,8 \mathrm{Aa}$ & $2,6 \mathrm{BC}$ & Trichodermil® & $2,7 \mathrm{Ba}$ & $3,1 \mathrm{ABa}$ & $2,4 \mathrm{CD}$ \\
\hline Stimucontrol® & $3,2 \mathrm{Aa}$ & $2,9 \mathrm{Ab}$ & $3,0 \mathrm{~A}$ & Stimucontrol® & $4,7 \mathrm{Aa}$ & $3,0 \mathrm{Ab}$ & $3,9 \mathrm{~A}$ \\
\hline Média & $2,7 \mathrm{a}$ & $2,6 a$ & & Média & $3,3 a$ & $2,5 b$ & \\
\hline $\mathrm{CV}(\%)$ & & 7,8 & & $\mathrm{CV}(\%)$ & & 16,54 & \\
\hline \multicolumn{4}{|c|}{ Massa fresca de plântula (mg) } & \multicolumn{4}{|c|}{ Massa seca de plântula (mg) } \\
\hline \multirow{2}{*}{ Produto } & Qualidac & semente & \multirow{2}{*}{ Média } & \multirow{2}{*}{ Produto } & \multicolumn{2}{|c|}{ Qualidade da semente } & \multirow{2}{*}{ Média } \\
\hline & $\mathrm{L} 1$ & L2 & & & $\mathrm{L} 1$ & $\mathrm{~L} 2$ & \\
\hline Testemunha & $90,0 \mathrm{Aa}$ & $250,0 \mathrm{Aa}$ & $170,0 \mathrm{~A}$ & Testemunha & $48,0 \mathrm{Aa}$ & $44,0 \mathrm{Aa}$ & $46,0 \mathrm{~A}$ \\
\hline Ecotrichß & $140,0 \mathrm{Aa}$ & $120,0 \mathrm{Aa}$ & $130,0 \mathrm{~A}$ & Ecotrich® & $46,0 \mathrm{Aa}$ & $46,0 \mathrm{Aa}$ & $46,0 \mathrm{~A}$ \\
\hline Quality $B$ & $300,0 \mathrm{Aa}$ & $320,0 \mathrm{Aa}$ & $310,0 \mathrm{~A}$ & Quality® & $44,0 \mathrm{Aa}$ & $36,0 \mathrm{ABa}$ & $40,0 \mathrm{AB}$ \\
\hline Trichodermil® & $220,0 \mathrm{Aa}$ & $170,0 \mathrm{Aa}$ & $190,0 \mathrm{~A}$ & Trichodermil® & $42,0 \mathrm{Aa}$ & $20,0 \mathrm{Bb}$ & $31,0 \mathrm{~B}$ \\
\hline Stimucontrol@ & $360,0 \mathrm{Aa}$ & $230,0 \mathrm{Aa}$ & $290,0 \mathrm{~A}$ & Stimucontrol® & $48,0 \mathrm{Aa}$ & $32,0 \mathrm{ABb}$ & $40,0 \mathrm{AB}$ \\
\hline Média & $224,0 \mathrm{a}$ & 219,0 a & & Média & $45,6 \mathrm{a}$ & $35,6 \mathrm{~b}$ & \\
\hline CV (\%) & & 74,22 & & CV (\%) & & 27,87 & \\
\hline
\end{tabular}

*Médias seguidas pelas mesmas letras, maiúsculas na coluna e minúsculas na linha, não diferem estatisticamente entre si pelo teste de Tukey a 5\% de probabilidade. L1- Lote 1; L2: Lote 2. 
Para as sementes do Lote 1, de alto vigor, a microbiolização com as formulações a base de Trichoderma não interferiu na resposta das variáveis de primeira contagem de germinação e germinação, porém ao analisar as sementes de baixo vigor (Lote 2) foi possível observar que Trichoderma spp. possibilitou incremento na germinação e primeira contagem destas sementes em relação ao tratamento testemunha. Carvalho et al. (2000) observaram que as melhores respostas a tratamentos que buscam a melhoria no desempenho de sementes são obtidas em sementes de baixa qualidade. Considerando que não se conhece a qualidade da maioria dos lotes de sementes de canola, a utilização da microbiolização pode proporcionar ganhos expressivos à cultura.

Nas avaliações do Lote 2, de baixo vigor, Trichoderma asperellum, base da formulação comercial do produto Quality® não alterou a germinação e primeira contagem, demonstrando que esta espécie do antagonista pode não ser benéfica para a germinação de sementes de canola, quando comparado com os resultados apresentados pelas formulações à base de T. harzianum, uma vez que os outros produtos proporcionaram incremento nestas variáveis.

Segundo Benítez et al. (2004) e Vinale et al. (2012), o aumento na produtividade de plantas é evidente quando as sementes são expostas a conídios de Trichoderma spp., porém quando separadas, sem contato direto com as sementes, apresentam aumento substancial em seu crescimento, o que sugere que Trichoderma spp. atua não somente como promotor de crescimento, mas também como molécula sinalizadora devido a sua produção de metabolitos. É importante salientar que os testes de vigor são complementares aos resultados do teste de germinação e são mais representativos em uma condição de campo, assim é importante realizar outros testes para que se tenha informações mais detalhadas sobre a qualidade das sementes. Portanto, tem-se a necessidade de maiores estudos, uma vez que estes não são padronizados para culturas como a da canola (Migliorini, 2012).

Para as variáveis comprimento de parte aérea e comprimento de raiz, em ambas as condições de qualidade das sementes, houve efeito dos tratamentos. $O$ produto Stimucontrol®, a base de T. harzianum, promoveu o crescimento da parte aérea das plântulas mesmo no lote de alto vigor. Já em condições de baixo vigor, todos os produtos à base de $T$. harzianum proporcionaram este estímulo e novamente, o produto Quality ${ }^{\circledR}$, à base de $T$. asperellum não diferiu da testemunha. Faria et al. (2003) observaram que plantas de algodoeiro oriundas de sementes tratadas com $T$. harzianum aumentaram satisfatoriamente a promoção do crescimento de plântulas, em relação a testemunha.

As sementes do Lote 2, de baixo vigor, quando microbiolizadas com Trichoderma spp. apresentaram maiores valores no comprimento de raízes comparado à testemunha. Porém, em alguns tratamentos de sementes do Lote 1, de alto vigor, houve redução do comprimento de raízes, contudo, não houve redução na massa fresca e seca, e, portanto, pode não representar redução de crescimento posterior. Em trabalho desenvolvido por Benítez et al. (2004), a promoção do crescimento e desenvolvimento do sistema radicular, resultando do tratamento de sementes com Trichoderma, foi atribuído à capacidade que o fungo possui em aumentar sua superfície total do sistema radicular, resultando assim em maior acesso aos elementos minerais.

Os mecanismos de ação dos fungos promotores do crescimento vegetal são específicos e podem variar conforme o ambiente, substrato, disponibilidade de nutrientes e ainda a interferência de outros microrganismos (Machado, 2015). Menten (1995) salienta que os efeitos do tratamento de sementes na germinação ocorrem a médio e longo prazo, como acontece, por exemplo, com a redução no avanço de desenvolvimento de doenças e/ou introdução de patógenos na área.

Há grande variabilidade entre os isolados de Trichoderma spp. utilizados nas formulações comerciais. Entre os produtos à base de Trichoderma, a espécie Trichoderma harzianum é a mais comercializada mundialmente, sendo encontrada, quando considerado os produtos sem mistura de microrganismos, em 38,8\% dos produtos comerciais. Porém, quando considerado os produtos onde há mistura de espécies de Trichoderma, este valor pode chegar a $50 \%$ dos produtos disponíveis no mercado mundial, e este valor sobe para $60 \%$ quando considerado todos os produtos, incluindo aqueles que espécies de Trichoderma são misturados com outros fungos, bactérias e micorrizas (Bettiol et al., 2019).

Diversos experimentos já conduzidos com o uso da técnica de microbiolização constataram que grande parte dos estudos são conduzidos com utilização de T. harzianum, o que possibilita concluir que seu uso é maior quando comparado a outras espécies do gênero, como T. asperellum.

\section{Conclusão}

A ação de Trichoderma spp. sobre o incremento na qualidade fisiológica de sementes de canola foi maior em sementes de baixo vigor.

Sementes de canola de baixo vigor, microbiolizadas com formulações comerciais a base de Trichoderma harzianum, apresentaram melhor qualidade fisiológica.

\section{Referências}

Asuming BS (2013) Phosphate solubilizing microorganisms and their ability to influence yield of rice. Agricultural Science Research Journal 3(12):379-386.

Àvila RM, Braccini LA, Scapim AC, Martorelli TD, Albrecht PL (2005) Testes de laboratório em sementes de canola e a correlação com a emergência de plântulas em campo. Revista Brasileira de Sementes 27(1):62-70. 
Benítez T, Rincón AM, Limón MC, Codon AC (2004) Biocontrol mechanisms of Trichoderma strains. International Microbiology 7(4):249-260.

Bettiol W, Pinto VZ, et al (2019) Produtos comerciais à base de Trichoderma. In: Meyer CM, Mazaro MS, Silva CJ (1) Trichoderma: uso na agricultura. Embrapa soja. p. $45-160$.

Brasil. Ministério da Agricultura, Pecuária e Abastecimento. Regras para Análise de Sementes. Brasília: Mapa/ACS, 2009. 395p.

Carvalho LF, Medeiro Filho S, Rossetti AG, Teófilo EM (2000) Condicionamento osmótico em sementes de sorgo. Revista Brasileira de Sementes 22(1):185-192.

Chagas LFB, Castro HG, Colonia BSO, Carvalho Filho MR, Miller LO, Chagas Junior AF (2016) Efficiency of Trichoderma spp. as a growth promoter of cowpea (Vigna unguiculata) and analysis of phosphate solubilization and indole acetic acid synthesis. Brazilian Journal of Botany 38(4):1-11.

Chagas LFB, Chagas Junior AF, Fidelis RR, Carvalho Filho MR, Miller LO (2017) Trichoderma asperellum efficiency in soybean yield components. Comunicata Scientiae 08(1):165-169.

Conab (2019) Acompanhamento da safra brasileira de grãos/ safra 2018/19. Disponível em: < https://www.conab.gov.br/info-agro/safras/graos/boletim-da-safra-degraos> (Acesso em 10 nov 2019)

Contreras CHA, Macías LR, Cortés CP, López JB (2009) Trichoderma virens a plant beneficial fungus, enhances biomass production and promotes lateral root growth through an auxin-dependent mechanism in Arabidopsis. Plant Physiology 149(3):1579-1592.

Ferreira DF (2008) SISVAR: Um programa para análises e ensino de estatística 6:36-41.

Faria KYA, Albuquerque CM, Neto CD (2003) Qualidade fisiológica de sementes de algodoeiro submetidas a tratamentos químico e biológico. Revista Brasileira de Sementes 25(1):121-127.

Harman GE (2000) Myth and dogmas of biocontrol changes in perceptions derived from research on Trichoderma harzianum T - 22. Plant Disease 84:377$-393$.

Junges E, Bastos BO, Toebe M, Muller J, Pedroso DC, Muniz MFB (2014) Restrição hídrica e peliculização na microbiolização de sementes de milho com Trichoderma spp.. Comunicata Scientiae 5(1):18-25.
Kapri A, Tewari L (2010) Phosphate solubilization potential and phosphatase activity of rhizospheric Trichoderma spp. Brazilian Journal Microbiology 41(3):787-795.

Machado DFM, Parzianello FR, Silva ACF, Antoniolli ZI (2012) Trichoderma no Brasil: $O$ fungo e o bioagente. Revista de Ciências Agrárias 35(1): 274-288.

Machado MFD, Tavares PA, Lopes JS, Silva Ferreira CA (2015) Trichoderma spp. na emergência e crescimento de mudas de Cambará (Gochnatia polymorpha (Less.) Cabrera). Revista Árvore 39(1):167-176.

Marcos Filho J (1999) Teste de envelhecimento acelerado. In: Krzyzanowski FC, Vieira RD, França Neto JB (Ed) Vigor de sementes: conceitos e testes. p.1-24.

Menten JOM (1995) Patógenos em Sementes: detecção, danos e controle químico, 1ํ Reimpr. São Paulo, Ciba Agro. $321 \mathrm{p}$.

Migliorin P, Kulczynk MS, Silva AT, Bellé C, Koch F (2012) Efeito do tratamento químico e biológico na qualidade fisiológica e sanitária de sementes de canola. Enciclopédia biosfera, Centro Científico Conhecer 8(15):788-801.

Oliveira AG, Chagas Júnior AF, Santos GR, Miller LO, Chagas LFB (2012) Potencial de solubilização de fosfato e produção de AIA por Trichoderma spp.. Revista Verde de Agroecologia e Desenvolvimento Sustentável 7(3):149-155.

Santos CC, Oliveira FA, Santos MS, Talamini V, Ferreira JMS, Santos FJ (2012) Influência de Trichoderma spp. sobre o crescimento micelial de Thielaviopsis paradoxa. Scientia Plena 8(4):1-5.

Silva JC, Torres DB, Lustosa DC, Filippi MCC, Silva GB (2012) Rice sheath blight biocontrol and growth promotion by Trichoderma isolates from the Amazon. Amazonian Journal of Agricultural and Environmental Sciences 55(4):243-250.

Tomm GO (2007) Indicativos tecnológicos para produção de canola no Rio Grande do Sul. Embrapa Trigo, Passo Fundo - RS.

USDA - United States Department of Agriculture (2019) Oilseeds: World markets and trades Disponível em: $<$ https://apps.fas.usda.gov/psdonline/circulars/oilseeds. pdf $>$ (Acesso em 10 nov 2019).

Vinale F, Sivasithamparam K, Ghisalberti EL, Ruocco M, Wood S, Lorito M (2012) Trichoderma secondary metabolites that affect plant metabolism. Natural Product Communications 7(11):1545-1550. 